\title{
PERFIL DE UTILIZACIÓN DE MEDICAMENTOS ADMINISTRADOS POR SONDA ENTERAL EN EL HOSPITAL UNIVERSITARIO
}

\section{PROFILE OF THE USE OF DRUGS ADMINISTERED VIA ENTERAL FEEDING TUBE IN A UNIVERSITY HOSPITAL}

\author{
Verena Gabriela do Ouro Reis (1), Márcia Ferreira Candido (2), \\ Rosângela Passos de Jesus (3), Raquel Simões Mendes-Netto (2) \\ (1) Departamento de Fisiologia da Universidade Federal de Sergipe (UFS). Brasil \\ (2) Núcleo de Nutrição da Universidade Federal de Sergipe. Brasil \\ (3) Escola de Nutrição da Universidade Federal da Bahia. Brasil
}

\begin{abstract}
The objective of this study was to evaluate the conditions of use of medicines in patients receiving enteral nutrition at units of University Hospital. The data collection was done using a structured questionnaire, based on patient's clinical files and observation of the nurse aides' routine in the period between January and April of 2009. Results demonstrated that $16.7 \%$ of the patients needed a feeding tube replacement because of obstructions. Approximately $52 \%$ of prescribed medicines were in solid form and at least eight medicines administered via feeding tube were recognized to be capable of drug-nutrient interaction in enteral feeding and affected at least one patient each. A conclusion can be drawn that there were prescription errors and inadequacy in techniques of administration of medicines to patients using probes of enteral nutrition.
\end{abstract}

Key words: Pharmaceuticals, enteral, drug-enteral nutrition interaction, derivation of pharmaceuticals via nasogastric tube.

Este trabajo fue recibido el 2 de Junio de 2010 y aceptado para ser publicado el 20 de Agosto de 2010.

\section{INTRODUCCIÓN}

La alimentación enteral se refiere a la administración de nutrientes en formulaciones líquidas por medio de un catéter introducido oro o nasoenteral para pacientes imposibilitados de ingerir oralmente los alimentos, con el propósito de restaurar el estado nutricional $(1,2)$.

La elección de la terapia nutricional a realizar, tanto como su vía de administración, debe considerar el cuadro clínico y las condiciones generales del paciente. Deben ser evaluados individualmente, con base a la evolución clínica, condiciones funcionales del trato digestivo, características físicas y químicas de la fórmula, proporción de macronutrientes, necesidades metabólicas específicas y costo beneficio (3).

Las dietas enterales pueden administrarse por infusión en bolus vía jeringuilla, goteo intermitente por gravedad o goteo continuo con auxilio de bomba de infusión. Para definir el mejor método de infusión, el equipo debe basarse en el estado clínico del paciente, en el objetivo de la terapia y en la calidad de vida (1). La terapia nutricional enteral puede ser administrada vía sonda nasoenteral, nasogástrica o por sondas de gastrostomía, duodenostomía o yeyunostomía (2).

Siempre que es posible, las sondas nasoenterales (SNE) o nasogástricas (SNG) son las más utilizadas en la práctica clínica, porque son de fácil manoseo y menos costosas, además que presentan menos riesgos de complicaciones. Sin embargo, si la indicación de la SNE es superior a 30 días, se recomienda la confección de una ostomía para reducir los riesgos de la permanencia prolongada del catéter nasoenteral (4).

A pesar de las buenas prácticas de administración de terapia nutricional enteral (TNE), no validar la utilización de la misma sonda de alimentación para administración de medicamentos, la enorme disponibilidad de drogas para administración oral, los bajos costos y el menor riesgo de complicaciones en comparación con las drogas intravenosas, justifican el 
uso de esas drogas vía sondas de alimentación (5). Sin embargo, los medicamentos usados oralmente, a pesar de los muchos avances farmacéuticos y terapéuticos, no son diseñados, testados, rotulados o aprobados por el fabricante o FDA (Food and Drug Administration) para el uso en sistema de nutrición (6). De esta forma, los pacientes que reciben nutrición por sonda enteral y algún tratamiento médico asociado presentan un riesgo adicional.

La asociación de dietas enterales y medicamentos envuelve aproximadamente al 51,5\% de las ocurrencias de complicaciones en la práctica clínica, aunque todavía hay poca información disponible sobre las posibles interacciones entre las formulaciones enterales y los diversos productos farmacéuticos en el mercado (7).

Un estudio clínico demostró que cerca del $40 \%$ de los pacientes evaluados tenían más de cinco medicamentos administrados por SNE, lo cual es considerado polifarmacia, y más de trece administraciones por día5. Las alteraciones debido a esas asociaciones son conocidas como incompatibilidades, y pueden ser clasificadas en física, farmacéutica, farmacológica, farmacocinética y fisiológica. Formación de gel, separación de fases y alteración de consistencia y viscosidad son los ejemplos más comunes de incompatibilidad física, siendo esa las que más trastornos causan al paciente, porque provocan obstrucción de la luz de la sonda con consecuente necesidad de sustitución del catéter (8).

Según Dickerson (2004), algunas precauciones pueden tomarse con el fin de evitar los tipos de incompatibilidades: preferir la administración oral siempre que sea posible; lavar la sonda con agua antes y después de la administración del medicamento; si el medicamento contiene una cantidad sustancial de sorbitol o está en forma de comprimido revestido, una forma alternativa debe ser considerada. Adicionalmente, el autor sugiere que los comprimidos de acción rápida deben ser convertidos en un polvo fino y acrecentados con agua para formar una mezcla de pasta fluida antes de la administración por la sonda enteral; si no hay otra forma farmacéutica alternativa, los medicamentos en cápsula de gelatina blanda deberá aspirarse su contenido con una jeringuilla y mezclado con agua, debido a su viscosidad, para después ser administrado; las cápsulas de acción inmediata deben ser abiertas y mezcladas con agua para formar una pasta fluida (9).

De acuerdo a Chan (2002), en caso que no se tomen esos cuidados, posiblemente ocurrirán interacciones que afectarán la absorción de medicamentos y/o nutrientes administrados por la vía oral o enteral, causando aumento o disminución de la biodisponibilidad de los mismos (10).
Las condiciones de la administración de medicamentos por sonda enteral se presentan a veces en situaciones más allá de lo esperado. Un estudio realizado en dos hospitales universitarios de Holanda mostró que $76 \%$ de las administraciones conducidas por los enfermeros presentan errores, desde el no lavado de la sonda antes y después de las medicaciones, hasta la trituración de medicamentos que no deben pasar por ese proceso mecánico (11).

Considerando el gran número de pacientes que reciben medicamentos por sondas enterales y la falta de conocimiento específico sobre el manejo adecuado de estas, el objetivo del presente estudio fue describir el perfil general de utilización de fármacos en pacientes que usan una sonda de nutrición enteral en un hospital universitario del nordeste de Brasil.

\section{MATERIAL Y MÉTODO}

Considerando la falta de información en nuestro medio sobre prevalencia y característica de las prescripciones de medicamentos por la vía de nutrición enteral, se optó por el estudio descriptivo, exploratorio, de campo y con enfoque cualitativo, realizado en el Hospital Universitario (HU) de la Universidad Federal de Sergipe (UFS). La muestra fue seleccionada por conveniencia (no probabilístico), siendo un paso inicial para futuros estudios.

El presente estudio se realizó en base a la recolección de información a partir de las fichas médicas (ficha de evolución médica, ficha de prescripción y ficha de evolución de enfermería) y mapa de prescripciones dietoterápicas de todos los pacientes que hicieron uso de la sonda de nutrición enteral (SNE) en el periodo en que estuvieron internados en el HU-UFS, de enero a abril del año 2009.

Las informaciones fueron recogidas en un formulario estructurado que contenía las variables de interés para el estudio, a) en relación al paciente: género, edad, clínica de internación, diagnóstico y desenlace clínico hospitalario; b) en relación al medicamento: nombre de los medicamentos prescritos por la vía de la sonda nasoenteral, posología, forma farmacéutica, vía de administración, inicio y término de la prescripción; c) en relación a la terapia nutricional: fecha de inicio y término de la terapia por SNE, fórmula prescrita y número de sustitución de los catéteres.

Para analizar la edad, los pacientes se agruparon en tres categorías, de acuerdo a la clasificación de la Organización Mundial de la Salud (OMS): de 0 a 18 años como niños y adolescentes, de 19 a 64 años como adultos, y más de 65 años como adultos mayores.

En el análisis del número de medicamentos pres- 
critos y administrados se consideró la clasificación de Linjakumpu y col. (12). Esta clasificación conceptúa polifarmacia como la utilización simultánea de más de cinco medicamentos durante el tratamiento.

Para la identificación de las sustancias de cada fármaco prescrito a partir de los nombres comerciales fue utilizado el Diccionario Terapéutico Guanabara (13) y la lista de medicamentos genéricos disponible en el sitio de Anvisa (14).

\section{Evaluación del uso de medicamentos por SNE}

Se utilizó como criterio de evaluación del uso de medicamentos por la vía SNE las recomendaciones del Servicio de Información sobre Fármacos de los Estados Unidos $(15,16)$. En base a estas recomendaciones se buscó categorizar como adecuada o inadecuada la prescripción, derivación y administración de medicamentos en cada clínica del hospital (clínica médica, pediátrica, quirúrgica, psiquiátrica y UTI).

La prescripción médica de fármacos para administración por SNE fue evaluada sobre la mejor forma farmacéutica para administración por sonda, disponibilidad de otra forma farmacéutica en el hospital además de la prescrita y la posibilidad de administración por otra vía a no ser la sonda nasoenteral.

Para esta evaluación se utilizó también la Lista de Padronización de Medicamentos disponible en la Unidad Hospitalaria, destinada a uniformizar los fármacos y las formas farmacéuticas prescritas.

La derivación del fármaco fue evaluada por medio de datos sobre las técnicas utilizadas: forma de trituración, de dilución, tipo de agua y materiales usados. La administración fue analizada de acuerdo con datos sobre adición del medicamento al frasco de dieta enteral, lavado de la sonda e interrupción del suministro de la dieta antes y después del fármaco ser administrado.

Las informaciones sobre la preparación y administración de medicamentos por sonda de nutrición enteral fueron obtenidas por medio de la observación de la rutina de los técnicos en enfermería. Cada clínica fue seguida en el período de un mes, en días y horarios variados, para tener un mayor alcance de los auxiliares de enfermería. Como el equipo de auxiliares es muy extenso, fueron obtenidos datos complementarios por testimonios y relatos de los mismos sobre los métodos de derivación farmacéutica, tratando de identificar los principales problemas asociados a la sonda enteral.

\section{Tratamiento de los datos}

El análisis de los datos fue de carácter descriptivo, los resultados fueron sintetizados en la forma de cuadros y tablas para la interpretación y análisis de las variables y categorías estudiadas, presentadas en frecuencia absoluta y relativa. Para la tabulación de los datos se utilizaron planillas del programa Microsoft Excel versión 2007.

\section{Aspectos éticos}

Antes de recolectar los datos se contactó a la dirección del hospital y con el equipo multidisciplinario comprometido en el tratamiento de los pacientes, para esclarecer y conseguir autorización para los procedimientos a ser realizados y los objetivos del estudio. El presente estudio fue aprobado por el Comité de Ética e Investigación del Hospital Universitario de la UFS (CAAE 1101.0.000.107-8).

\section{RESULTADOS}

El análisis de los datos de ese trabajo permitió la obtención del perfil de los pacientes que utilizaron sonda de nutrición enteral, como se muestra en la tabla 1. De los 24 pacientes internados, la mayoría eran adultos $(54,2 \%)$, de género femenino $(58,4 \%)$ y la UTI fue la clínica de internación donde hubo el mayor número de pacientes con SNE (37,5\%), seguido de pediatría $(29,2 \%)$. Se analizó también el desenlace clínico de la internación hospitalaria de los pacientes, de los cuales aproximadamente $38,0 \%$ fueron óbito.

Aproximadamente $62,5 \%$ de los pacientes usaron la sonda de nutrición enteral por más de diez días, y cerca de 42,0\% tuvieron más de cinco drogas prescritas por la vía de sonda enteral. La frecuencia de administración de fármacos por la vía SNE por día fue de $25,0 \%$ en la clasificación de pacientes con más de trece administraciones por día. Fue necesario el cambio de sonda en cuatro $(16,7 \%)$ pacientes.

En el presente estudio los medicamentos más prescritos fueron: aceite mineral dimeticona, dipirona sódica y paracetamol, todos en forma farmacéutica líquida. En relación con los medicamentos más prescritos en la forma sólida, los más frecuentes fueron: ácido fólico, pirimetamina y sulfadiazina.

Fueron identificados 52 medicamentos prescritos, 27 en forma sólida y 25 en forma líquida (tabla 2). De los medicamentos prescritos, el clonazepam y haloperidol se presentaron tanto en forma sólida como en forma líquida. Con relación a la evaluación de la prescripción médica se identificó que aproximadamente $35 \%$ de las prescripciones fueron inadecuadas.

En la tabla 3 se describen los medicamentos que fueron identificados en las prescripciones médicas evaluadas, con reconocida interacción droga-nutrición enteral y el número de pacientes afectados en el presente estudio. 


\section{DISCUSIÓN}

Antes de proceder a discutir los resultados, es necesario recordar que el estudio empleó un proceso de muestra por conveniencia. Por lo tanto, lo datos se refieren a grupos específicos de pacientes y no pueden se generalizados a toda la población de pacientes hospitalizados en Sergipe. Sin embargo, nuestros resultados nos ayudan a entender la complejidad de la terapia de nutrición enteral en los hospitales los que fueron semejantes a los encontrados en otras investigaciones $(5,16)$.

\section{TABLA 1}

\section{Características de los pacientes en SNE internados en el HU-UFS}

Variable

$\mathbf{N}^{\circ}$ total de pacientes

Género:

Femenino

Masculino

Edad (años):

0 a 18

19 a 65

$>65$

Clínicas de internación:

UTI

Pediátrica

Cirugía

Psiquiátrica

Médica

Desenlace clínico:

Alta

Óbito

Días de uso de SNE:

$\leq 10$

$>10$

$\mathrm{N}^{\circ}$ de medicamentos prescritos por SNE:

$\leq 5$

$>5$

Administración de medicamentos por día:

$\leq 13$

$>13$

$\mathrm{N}^{\circ}$ de cambios de sonda:

Cero

$\geq 1$

Medicamentos líquidos más prescritos:

Aceite mineral

Dimeti

Dimeticona

Dipirona sódica

Paracetamol

Medicamentos sólidos más prescritos:

Ácido fólico

Pirimetamina

Sulfadiazina 
La caracterización de la utilización de la SNE en los pacientes estudiados, principalmente en lo que se refiere a los días de uso, número de medicamentos prescritos y número de administraciones hechas en el día, son indicados en la literatura como factores de riesgo que aumentan la posibilidad de complicaciones mecánicas con catéter de nutrición enteral, como la obstrucción $(5,17)$.

La principal consecuencia en relación a la obstrucción del catéter fue la necesidad de cambio de la sonda. Para el paciente significa un estrés físico intenso por la necesidad de volver a colocar la sonda, y puede debilitar aún más al paciente y aumentar las posibilidades de posicionamiento inadecuado de la misma, además de demasiada exposición a radiación para confirmación la posición del tubo. Para el hospital, esta necesidad de cambio representa un aumento de costos con el paciente durante el período de internación, dado que los catéteres enterales presentan un costo elevado. La reducción en el número y en la frecuencia de estas drogas pueden disminuir los cambios de sonda, y consecuentemente los problemas ya mencionados (18).

En el presente estudio el cambio de la sonda nasoenteral debido a obstrucciones fue confirmada en $16,7 \%$ de los pacientes, resultados similares a un trabajo clínico que demostró una frecuencia esperada para obstrucción de sonda de nutrición enteral causada por medicamentos en cerca de $15,0 \% 16$. Sin embargo, este número puede ser subestimado debido a la falta de registros de otros cambios en los prontuarios clínicos en el hospital analizado. De los cuatro pacientes en quienes el cambio por obstrucción fue confirmado, tres presentaron más de cinco medicamentos por SNE, más de 13 administraciones por día y hacían uso de la nutrición enteral por un periodo superior a diez días. El cuarto paciente tenía apenas un medicamento prescrito vía SNE: poliestirenosulfonato de calcio (Sorcal $®$ ), no obstante, tal vez la forma de presentación en polvo oral haya sido el factor responsable por la obstrucción (19).

La tabla 2 presenta la prescripción médica de los fármacos por vía SNE; de los 52 medicamentos prescritos, $34,6 \%$ presentaron una inadecuada prescripción, siendo el principal error la indicación de comprimidos con revestimiento en $38,9 \%$. El segundo error más frecuente fue la indicación de la forma sólida, incluso estando disponible en el hospital en forma farmacéutica líquida en $33,3 \%$ de los casos. La selección de la droga inapropiada para uso por vía de la sonda puede causar una potencial toxicidad, reducción de la eficacia y obstrucción del tubo. Las recomendaciones descritas por diversos autores orientan que en la prescripción de fármacos por vía de la sonda se debe reconocer los medicamentos usados por el paciente que están disponibles en forma líquida o que pueden ser triturados. Para los medicamentos que no pueden ser derivados, es necesario considerar otra vía de administración (transdérmica, rectal, parenteral), dependiendo del estado clínico y diagnóstico del paciente $(15,16)$. Finalmente, si no hubiera una forma alternativa del medicamento, otra droga con efecto farmacológico semejante en su forma líquida o pastilla simple debe ser considerada (11).

La mayor prevalencia de medicamentos líquidos administrados en el HU está relacionada al gran número de pacientes pediátricos que hacían uso de la nutrición enteral durante el estudio, ya que las formulaciones para estos pacientes están generalmente disponibles en forma de solución oral. De los 25 medicamentos prescritos en forma líquida, 17 fueron prescritos exclusivamente en el servicio de pediatría, representando $68 \%$ de los medicamentos líquidos utilizados. Excluyendo al srvicio de pediatría de la muestra del presente estudio, se percibe que existe una preferencia de prescripción de fármacos en la forma sólida, cerca del $80 \%$ del total de medicamentos prescritos. Estos resultados son equivalentes a otros estudios conducidos en Brasil, como el desarrollado por

\section{TABLA 2}

Características de los medicamentos prescritos por SNE en el HU-UFS

Variable

$\mathrm{N}^{\circ}$ total de medicamentos
$\mathbf{N}$

52

Forma farmacéutica:

Sólida

Líquida

Prescripción de medicamentos:

Adecuada

Inadecuada
27

25

34

18
$\%$

$51,9 \%$

$48,1 \%$

$65,4 \%$

$34.6 \%$ 
Heyneck (5) en pacientes adultos, que demostró que apenas $1 \%$ de los medicamentos más prescritos estaba en su forma farmacéutica líquida (solución oral o jarabe).

El tercer error de mayor prevalencia fue la prescripción de medicamentos hiperosmolares, lo que ocurrió en $27,8 \%$ de los casos. Muchos medicamentos líquidos tienen osmolaridad por encima de $1000 \mathrm{mOsm} / \mathrm{kg}$. Como la osmolaridad de las secreciones gástricas es de 100-400 $\mathrm{mOsm} / \mathrm{kg}$, pueden ocurrir diarreas, cólicos, distensión abdominal y vómitos cuando se administran medicamentos hiperosmolares en grandes cantidades por la vía del catéter para nutrición. Sin embargo, estos efectos pueden reducirse por dilución de esos medicamentos con 10 a $30 \mathrm{ml}$ de agua antes de su administración (15).

En el presente estudio se buscó también investigar los problemas relacionados con los procedimientos de derivación y administración de los medicamentos. En este aspecto fueron evaluados siete auxiliares de enfermería en sus actividades, en el referido hospital.

Para la derivación de las formas farmacéuticas sólidas, fueron observadas dos prácticas comunes entre los auxiliares de enfermería: trituración y posteriormente

\section{TABLA 3}

\section{Interacción fármaco-nutrición enteral.}

Fármaco Interacción

\section{Recomendaciones}

Pacientes

afectados
Ciprofloxacino

(comprimido o

suspensión oral)

Clorpromazina

(solución oral)

Fenitoína

(comprimido o

suspensión oral)

Hidróxido de

Aluminio

Lactulosa

(solución oral)

Aceite mineral

(solución oleosa)

\section{Omeprazol}

(cápsula)

Sulfato ferroso

(solución oral)
En contacto con los cationes metálicos da nutrición, hay reducción de la biodisponibilidad de la droga.

En contacto con la dieta hay precipitación y reducción de la biodisponibilidad de la droga.

Reducción drástica de la absorción (50-75\%) de la droga cuando es administrada junto a la nutrición enteral.

En contacto con la dieta hay precipitación y reducción de la biodisponibilidad de la droga.

En contacto con la dieta hay precipitación y reducción de la absorción de los nutrientes.

El aceite se adhiere a la sonda disminuyendo gradualmente su luz; el lavado con agua no minimiza la obstrucción porque tienen polaridades diferentes

Como la droga activa sufre degradación gástrica, es necesario una administración diferenciada.

Debido a su viscosidad hay mayor probabilidad de causar obstrucción de la sonda.
Interrumpir la nutrición enteral 2

horas antes y 4 horas después de la administración.

Interrumpir la nutrición enteral 1 hora antes y 2 horas después de la administración.

Interrumpir la dieta 2 horas antes y después de la administración y monitorear los niveles plasmáticos.

Interrumpir la nutrición enteral 1 hora antes y 2 horas después de la administración.

Evitar la administración de cantidades elevadas del fármaco.

Evitar la administración de ese tipo de laxante, acrecentando una dieta enteral con fibras.

Administrar el contenido de la cápsula mezclando el jugo de frutas ácidas (manzana, naranja), y hacer el lavado de la sonda con el mismo.

Lavar cuidadosamente la sonda antes y después de su administración. 
dilución, y la dilución directa del medicamento algunos minutos antes de la administración.

La trituración fue observada tanto para comprimidos simples como para grageas. El revestimiento entérico, presente en grageas, tiene la finalidad de hacer que la droga intacta traspase el estómago y llegue hasta el intestino donde será absorbida. Este tipo de formulación impide la destrucción de la droga por el ácido gástrico, reduce la irritación del TGI, y permite una acción retardada del medicamento. Además de eso, el procedimiento de trituración puede no ser completo, haciendo que sus partículas se agreguen cuando son humedecidas. Por lo tanto, la pérdida del revestimiento entérico y trituración del contenido de las grageas no debe ser indicada pues es grande el riesgo de ineficiencia del medicamento y obstrucción de la sonda (8).

El material adecuado para la trituración, grado y pistilo, solo fue encontrado en una clínica del hospital, en las demás la trituración era hecha en el propio blíster del medicamento o con auxilio de cualquier material resistente, como el mango de una tijera o la propia jeringuilla, para la homogenización. El agua usada era, en su gran mayoría, filtrada, lo que no es aconsejable, ya que los iones presentes en el agua pueden interactuar con algunos fármacos y así disminuir su biodisponibilidad (17).

La forma de cápsula fue poco prescrita para la derivación, no obstante, cuando fue utilizada, su procedimiento ocurrió de acuerdo con la literatura. La excepción se dio apenas para el omeprazol, que debe ser diluido con jugo de frutas ácidas (manzana, piña, naranja), para garantizar que la cantidad máxima de la droga llegue al duodeno, donde es absorbido. Después de la administración la sonda debe ser lavada como el mismo tipo de jugo (15).Todos los auxiliares preparan y administran solo un medicamento por vez, lo cual es lo indicado, pues evita posibles interferencias en el tratamiento terapéutico, al contrario del estudio publicado por Heydrich (17), donde fue demostrado que los auxiliares de enfermería realizaban la preparación de ocho medicamentos en un mismo recipiente y la administración de tal mezcla de una única vez al mismo paciente.

Los profesionales observados interrumpían el goteo de la nutrición enteral solo en el momento de la administración de los medicamentos. Ninguno interrumpía la dieta enteral por 30 minutos antes o después de la administración del fármaco, como se recomienda. Por otro lado, ningún profesional adicionó el medicamento directamente en la dieta enteral, práctica que no es recomendada debido al gran riesgo de incompatibilidad. Otra recomendación importante se refiere al lavado de la sonda entes y después de la administración del medicamento (9), no obstante, solo una auxiliar relató este procedimiento.

Los datos referentes a la evaluación de los auxiliares de enfermería pueden no ser representativos del hospital, sin embargo es un indicativo de consecuentes errores en la rutina hospitalaria. Existe la necesidad, entonces, de estudios futuros que evalúen, cuantitativamente, el grado de conocimiento sobre la derivación de medicamentos de todos los auxiliares de enfermería, con el objetivo de caracterizar mejor la adecuación de la derivación y administración de medicamentos.

Aunque en las últimas décadas los conocimientos y avances sobre la nutrición enteral hayan evolucionado, y asegurado mejores resultados terapéuticos, pocas informaciones están disponibles al respecto y probables interacciones entre las formulaciones entéricas y los innumerables medicamentos disponibles en el mercado (20). A pesar de la gran dificultad de comprobación de esas diferentes formas de interacción droga-nutriente, bien como colateralidad digestiva, la prescripción de muchos fármacos es desaconsejable para pacientes que hacen uso de terapia nutricional por vía de la sonda.

En el tabla 3 se observa que 17 pacientes tenían interacciones entre medicamentos y nutrientes en sus prescripción. Fue bastante prevalente la administración de el aceite mineral por vía de la sonda. Aproximadamente el $30 \%$ de los pacientes analizados fueron afectados por la interacción droga-nutriente de un único medicamento.

El consumo de aceite mineral interviene en la absorción de vitaminas liposolubles (A, D, E, K), Bcaroteno, calcio y fosfatos, debido a la barrera física y/o disminución del tiempo de tránsito intestinal $(21,22)$. El metabolismo de la vitamina $\mathrm{D}$, calcio y fosfatos está interrelacionado, o sea, la deficiencia de una de estas sustancias puede conducir a las anormalidades metabólicas, caracterizando como mala absorción secundaria (23). El uso excesivo de aceite mineral puede provocar raquitismo en los niños y osteomalacia en adultos, por deficiencia de calcio $(24,25)$.

Además, este medicamento se adhiere a la pared del catéter nasoenteral, reduciendo gradualmente la luz del tubo, favoreciendo la adhesión de residuos de la dieta, lo que aumenta el riesgo de obstrucción y, en consecuencia, cambio de la sonda (20).

En el estudio de Sánchez y col. (26), sobre la atención farmacéutica en pacientes con uso de SNE, la inserción del farmacéutico en la rutina diaria del equipo de terapia nutricional, presentó resultados muy satisfactorios. En solo un mes, de los 14 pacientes asistidos, 12 tuvieron intervenciones farmacéuticas realizadas en su tratamiento farmacológico. De estas, tres se relacionaban con el cambio de forma farmacéutica, tres sobre manipulación equivocada de comprimidos con revestimiento 
entérico y cápsulas, tres por interacción droga-nutrición enteral, una por interacción droga-droga y dos por administración equivocada de medicamentos con $\mathrm{pH}$ elevado.

\section{CONCLUSIONES}

A pesar de la amplia información que se ha obtenido en los últimos años sobre la administración de fármacos por sonda de nutrición enteral, todavía hoy ocurren fallas que pueden comprometer tanto la terapia nutricional como la eficacia del tratamiento farmacológico. Es importante considerar el estado nutricional del paciente, pues mientras peor es el estado nutricional, mayores son las interacciones, lo que puede resultar en superestimación o subestimación de la retención de nutrientes y error en las prescripciones de la dieta o respuesta terapéutica inesperada, dificultando la recuperación del paciente.

El presente estudio muestra que existe una diferencia entre el conocimiento de la práctica recomendada por la literatura y la práctica clínica usual. Por eso, los resultados obtenidos pueden subsidiar el proceso de educación permanente para todos los profesionales de la salud, propiciando mejorías en el tratamiento de los pacientes en nutrición enteral hospitalarios.

Para prevenir los problemas relacionados y la obstrucción por fármacos por medio de sondas, es necesario estimular estudios, principalmente sobre la relación de técnicas inadecuadas de administración y obstrucción de las sondas. La elaboración de una guía de preparación y administración de medicamentos vía SNE o Procedimiento Operacional Padrón (POP) puede contribuir en la utilización racional de los medicamentos, ayudando en la adecuada selección de la forma farmacéutica y de la técnica de derivación y administración. Además, se hace necesario una capacitación previa de los profesionales de salud que preparan y administran esos medicamentos, para asegurar la eficacia del tratamiento, prevenir riesgos mayores al paciente como la desnutrición intrahospitalaria y evitar gastos hospitalarios innecesarios.

\section{RESUMEN}

El presente estudio tuvo como objetivo describir el perfil general de utilización de fármacos en pacientes que usan una sonda de nutrición enteral en un hospital universitario. La recolección de información fue hecha por medio de un formulario estructurado a partir de las fichas clínicas de pacientes internados en el hospital y de la observación de la rutina de los auxiliares de enfermería, en el periodo de enero a abril de 2009. Los resultados demuestran que $16,7 \%$ de los pacientes necesitaron cambio de catéter para nutrición enteral, debido a obstrucción. Aproximadamente 52,0\% de los medicamentos prescritos estaban en la forma farmacéutica sólida y por lo menos ocho medicamentos administrados por sonda fueron reconocidos como capaces de provocar interacción fármaco-nutrición enteral, afectando por lo menos a un paciente. Se concluye que hay errores en la medicación y en las técnicas de administración de medicamentos para la sonda enteral.

Palabras clave: Fármacos, enteral, interacción fármaco-nutrición enteral, derivación de fármacos vía SNE.

Dirigir la correspondencia a:

Profesora

Raquel Simões Mendes-Netto

Núcleo de Nutrição

Centro de Ciências Biológicas e da Saúde

Universidade Federal de Sergipe.

Av. Marechal Rondon, s/n Jardim Rosa Elze

CEP 49100-000 Cidade Universitária

Professor "José Aloísio de Campos"

Universidade Federal de Sergipe

São Cristóvão-SE - Brasil

Teléfono: 7921056574

Fax: 7921056567

E-mail:raquel@ufs.br

\section{BIBLIOGRAFÍA}

1. Bloch AS, Mueller C. Suportes Nutricionais Enteral e Parenteral. In: Mahan LK, Escott-Stump S. Krause, 11nd ed. Alimentos, Nutrição \& Dietoterapia. São Paulo: Roca; 2005. p.510-31.

2. Shike M. Alimentação Enteral. In: Shils EM, Olson AJ, Shike M, Ross CA. Tratado de Nutrição Moderna na Saúde e na Doença. 2nd ed. São Paulo: Manole, 2003; p.1761-1775.

3. Cortes JFF, Fernandes SL, Nogueira-Maduro IPN, Basile-Filho A, Suen VMM, Santos JE, Vannuchih \& Marchini JS. Terapia nutricional no paciente criticamente enfermo. Medicina, Ribeirão Preto 2003; 36: 394-8.

4. Waitzberg DL, Fadul RA, Aanholt DPJV, Plopper C, Terra RM. Indicações e Técnicas de Ministração em Nutrição Enteral. In: Waitzberg DL. Nutrição Oral, Enteral e Parenteral na Prática Clínica. 2nd ed. São Paulo: Atheneu; 2005; p.561-571.

5. Heineck I, Bueno D, Heydrich J. Study on the use of drugs in patients with enteral feeding tubes. Pharm World Sci 2009; 31(2): 145-8.

6. Fortes ST, Silva MLT. Influências de Drogas no Metabolismo Nutricional. In: Waitzberg DL. Nutrição Oral, Enteral e Parenteral na Prática Clínica. 2nd ed. São Paulo: Atheneu; 2005; p. 915-23.

7. Seifert CF, Johnston BA, Rojas-Fernandes C. Drug administration through enteral feeding catheters. Am 
J Health Syst Pharm 2002; 59 (4): 378-9.

8. Gastaldi M, Silveira DSG. Ministração e Compatibilidade de Drogas em Nutrição Enteral. In: Waitzberg DL. Nutrição Oral, Enteral e Parenteral na Prática Clínica. 2nd ed. São Paulo: Atheneu; 2005; p.865-73.

9. Dickerson RN. Medication Administration Considerations for Patients Receiving Enteral Tube Feedings. Hosp Pharm 2004; 39(1): 84-9.

10. Chan LN. Drug-nutrient interaction in clinical nutrition. Clin Nut Metab Care 2002; 5: 327-32.

11. Van Den Bemt PMLA, Cusell MBI, Overbeeke PW, Trommelen M, Doorem D, Opohorst WR, Egberts ACG. Quality improvement of oral medication administration in patients with enteral feeding tubes. Qual Saf Health Care 2006; 15: 44-47.

12. Linjakumpu T, Hartikainen S, Klaukka T, Veijola J. Use of medications and polypharmacy are incresing among the elderly. J Clin Epidemiol. 2002; 55(8): 809-817.

13. Korolkovas A, França FAC. Dicionário Terapêutico Guanabara. 15nd ed. Rio de Janeiro: Guanabara; 2008.

14. Agência Nacional de Vigilância Sanitária. Lista de Medicamentos de Referência Brasil, 2009 Mar. Disponible en: hlttp://www.anvisa.gov.br/.[Consultada em 04 mai 2009].

15. Beckwith MC, Feddema SS, Barton RG, Graves C. A guide to drug therapy in patients with enteral feeding tubes: Dosage form selection and administration methods. Hosp Pharm 2004; 39(3): 225-237.

16. Wyman M. Medication Administration Through Enteral Feeding Tubes Pharmac Update 2008; 11(3) Disponible en h h $\mathrm{tp} / / \mathrm{www}$ clevelandclinicmeded. com/medicalpubs/pharmacy/. [Consultada em 6 jun 2009].

17. Heydrich J. Padrão de prescrição, preparo e administração de medicamentos em usuários de sondas de nutrição enteral internados em um hospital universitário [dissertation]. Porto Alegre (RS) Universidade
Federal do Rio Grande do Sul; 2006.

18. Bates DW, Spell N, Cullen DJ, Burdick E, Laird N, Petersen LA, et al. The costs of adverse drug events. J Am Med Assoc 1997; 277(4): 307-11.

19. Loureiro AAC, Ianhez LE, Sabbaga E. Avaliação Clínica de Resina Troca Cátions (calcium serdolit) no Tratamento e Prevenção da Hiperpotassemia. Rev Hosp Clin Fac Méd 1972; 27: 267-72.

20. Reis NT. Nutrição Clínica: Interações. 1nd ed. Rio de Janeiro: Rubio; 2004, p.37-93.

21. Clark JH, Russel GJ, Fitzgerald JF, Nagamori KE. Serum beta-carotene, retinol, and alpha-tocopherol levels during mineral oil therapy for constipation. Am J Dis Child, Chicago, 1987; 141(11): 1210-2.

22. Trovato A, Nuhlicek DN, Midtling JE. Drug-nutrient interactions. Am Fam Physician, Kansas City MO 1991; 44(5): 1651-8.

23. Yamreudeewong W, Henann NE, Fazio A, Lower DL, Cassidy T. Drug-food interaction in clinical practice. J Fam Pract, East Norwalk CT, 1995; 40(4): 376-84.

24. Roe DA. Drugs, diets and nutrition. Am Pharm, Washington DC 1978; 18(10): 62-64.

25. Insogna KL, Bordley DR., Caro JE, Lockwood DH. Osteomalacia and weakness from excessive antacid ingestion. J Am Med Assoc, Chicago, 1980; 244(22): 2544-6.

26. Sánches AIG, Almagro CGM, Aranzana C, Continente AC, Hernandez MAC. Atención farmacéutica en pacientes con nutricion enteral. Farm Hosp. 2006; 30(1): 44-8.

\section{ABREVIATURAS}

SNE - Sonda nasoenterales

SNG - Sonda nasogástricas

TNE - Terapia Nutricional Enteral

HU - Hospital Universitário

UFS - Universidad Federal de Sergipe

OMS - Organización Mundial de la Salud 